\title{
Research Article \\ Simulation of Octopus Arm Based on Coupled CPGs
}

\author{
Juan Tian and Qiang Lu \\ College of Information and Engineering, Taishan Medical University, Taian 271016, China \\ Correspondence should be addressed to Juan Tian; jtian@tsmc.edu.cn
}

Received 9 January 2015; Revised 25 March 2015; Accepted 5 May 2015

Academic Editor: Nan Xiao

Copyright (C) 2015 J. Tian and Q. Lu. This is an open access article distributed under the Creative Commons Attribution License, which permits unrestricted use, distribution, and reproduction in any medium, provided the original work is properly cited.

\begin{abstract}
The octopus arm has attracted many researchers' interests and became a research hot spot because of its amazing features. Several dynamic models inspired by an octopus arm are presented to realize the structure with a large number of degrees of freedom. The octopus arm is made of a soft material introducing high-dimensionality, nonlinearity, and elasticity, which makes the octopus arm difficult to control. In this paper, three coupled central pattern generators (CPGs) are built and a 2-dimensional dynamic model of the octopus arm is presented to explore possible strategies of the octopus movement control. And the CPGs' signals treated as activation are added on the ventral, dorsal, and transversal sides, respectively. The effects of the octopus arm are discussed when the parameters of the CPGs are changed. Simulations show that the octopus arm movements are mainly determined by the shapes of three CPGs' phase diagrams. Therefore, some locomotion modes are supposed to be embedded in the neuromuscular system of the octopus arm. And the octopus arm movements can be achieved by modulating the parameters of the CPGs. The results are beneficial for researchers to understand the octopus movement further.
\end{abstract}

\section{Introduction}

Animals exploit soft structures to move effectively in complex natural environments and the typical one is octopus [1]. Octopus whose body and arms totally lack hard elements is unique marine invertebrate. And its advanced motor skills and intelligent behavior have attracted interest from both biologists and roboticists [2,3]. Octopus arms have peculiar features such as the ability to bend in all directions, to achieve significant elongation, and to vary and control their stiffness [4].

Inspired by an octopus arm, the concept of continuum arms for use in robotic systems has been proposed and studied. Continuum arms have a large number of actuated degrees of freedom (DOF) and are therefore well suited for operations in highly constrained environments [5]. There have been several attempts to dynamically model continuum arms. Some dynamic models [5-8] inspired by the octopus arms are presented to explore possible strategies of movement control in the muscular hydrostat. However, it is difficult to control octopus arms with conventional techniques because of their high-dimensional body structures and their diverse body dynamics [9]. It is well known that the nervous system of the octopus is highly distributed throughout the entire body. The octopus has a relatively small central brain which controls the large peripheral nervous system of the arms. A typical example showing the effectiveness of this distribution of the nervous system is the reaching behavior [10-13]. Sumbre et al. [13] showed that the arm extensions can be evoked in arms whose connection with the brain has been severed. Because the evoked motions in denervated octopus arms were identical to natural bend propagations, an underlying motor program appears to be embedded in the neuromuscular system of the arm, which does not require continuous central control. And the researchers suggested that the major part of the voluntary movement is controlled by a pattern generator that is confined to the arm's neuromuscular system.

The central pattern generator (CPG) is neural circuit found in both invertebrate and vertebrate animals that can produce rhythmic patterns of neural activity without receiving rhythmic inputs [14-16]. The famous one is the Matsuoka model $[17,18]$. The CPG presents several interesting properties including distributed control, the ability to deal with redundancies, fast control loops, and allowing modulation of locomotion by simple control signals. 


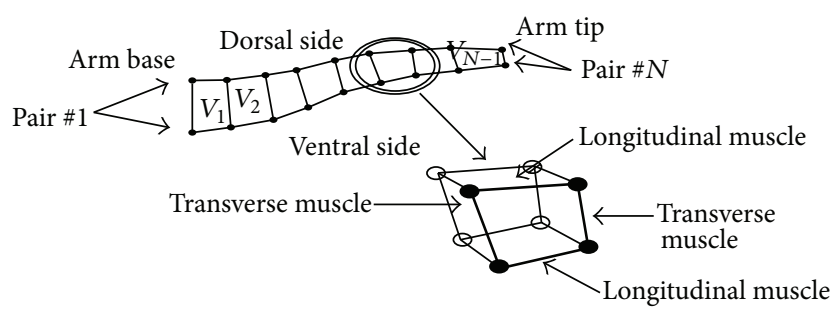

FIGURE 1: The octopus arm model.

In this paper, a model which includes three coupled central pattern generators (CPGs) is established and a 2-dimensional dynamic model $[10,19]$ of the octopus arm is used to explore possible strategies of movement control. And the CPG signals treated as activation are added on the ventral, dorsal, and transversal sides, respectively. The effects of the octopus arm are discussed using simulation method [20] when the parameters of the CPG are changed.

This paper is organized as follows. In Section 2, the 2dimensional dynamic model and the CPG model are presented. Simulation is shown in Section 3. The conclusions and future works are made in Section 4.

\section{The Octopus Arm Model and the CPG Model}

2.1. Structure of the Octopus Model. In this paper, the octopus arm model is a 2-dimensional dynamic model of a soft robotic arm $[10,19]$, utilizing only masses and springs for its dynamic characteristics. The arm is divided into $(N-1)$ rectangular segments and each one is defined by four vertices. For simplicity, the muscles are deprived of their mass and the entire arm's mass content is concentrated in point masses. The point masses are located in the four vertices of each segment, giving a total of $2 \mathrm{~N}$ masses. The idealized massless springs function as muscles and connect all the adjacent point mass pairs of the model. The $2 N$ masses are arranged in $N$ pairs, each consisting of one ventral and one dorsal mass. $(N-1)$ ventral and $(N-1)$ dorsal longitudinal muscles connect the $N$ ventral and $N$ dorsal masses, respectively. In addition, a transverse muscle connects each ventral-dorsal pair. In this paper, there are 10 segments in this model. Figure 1 shows the general structure of the modeled arm.

As a model of a muscular hydrostat, this model relies on a basic assumption that the muscle tissue of the octopus arm is incompressible. From this assumption, it is evident that the arm's volume must be constant at all times. Due to the constant volume constraint, a contraction of a muscle reduces its length in one axis but must increase its length in at least one of the axes perpendicular to the first. Therefore, in the 2-dimensional model, shortening a segment in one direction will force it to elongate in the other. Using this simple physical mechanism, the octopus arm gains almost unconstrained motion and transfers force from one direction to another without needing a rigid skeleton.
2.2. Dynamics of the Octopus Model. The basic model is 2dimensional, meaning that all the forces in its scope are vectors in an $x-y$ plane. Thus, the motion of the arm is constrained to a plain as well. The model takes into account four types of forces acting on the arm. The first is internal forces generated by the arm's muscles $\left(F^{m}\right)$. The second one is vertical forces caused by the combined influence of gravity and buoyancy $\left(F^{g}\right)$. The third one is drag force produced by the arm's motion through the surrounding medium $\left(F^{w}\right)$. The fourth is internal forces that maintain the constant volume constraint $\left(F^{c}\right)[10]$.

The motion equations can be written as

$$
M \ddot{q}=F^{m}+F^{g}+F^{w}+F^{c},
$$

where $M$ is a diagonal mass matrix and $\ddot{q}$ is the position vector.

A muscle is simulated by an ideal damped spring which exerts force caused by changes in its spring constant. The adjustments of the spring constant enable the user of the model to control the arm's movement. The arm's weight and the drag forces are calculated using the relevant physical theories. These forces are calculated from algebraic and differential manipulations on the equations of motion and the volume constraint.

There are two types of muscle models: one is the nonlinear muscle model and the other is linear damped spring model [10]. In this paper, every linear muscle in the linear model exerts the following force:

$$
f(t)=\left[k_{0}+k_{\max } a(t)\right]\left[l(t)-l_{\text {rest }}\right]+\alpha \frac{d l(t)}{d t}
$$

where $l_{\text {rest }}$ is the rest length of the muscle. This was chosen as the largest length at which both active and passive forces are zero in real muscles. The linear damping coefficient $\alpha$ has dimensions of $\mathrm{Ns} / \mathrm{m}$. The passive spring constant of the muscle is expressed by $k_{0}$ and the maximal active spring constant of the muscle by $k_{\max }$, both having dimensions of $\mathrm{N} / \mathrm{m} . a(t)$ is a dimensionless activation function.

\subsection{Implementation and Parameters of the Octopus Model.} The model enables the user to activate the arm by changing the constants of the muscle simulating springs. Any number of spring constants can be changed simultaneously and a given set of different spring constants changes is dubbed activation. All the activations last a constant time. The simulation recalculates the coordinates and velocities for each simulation time interval and changes the activation for each activation time interval. All the parameters set either the physical environment of the arm or various technical features of the simulation. Table 1 summarizes some of the more influential parameters of the simulation. 




(a)

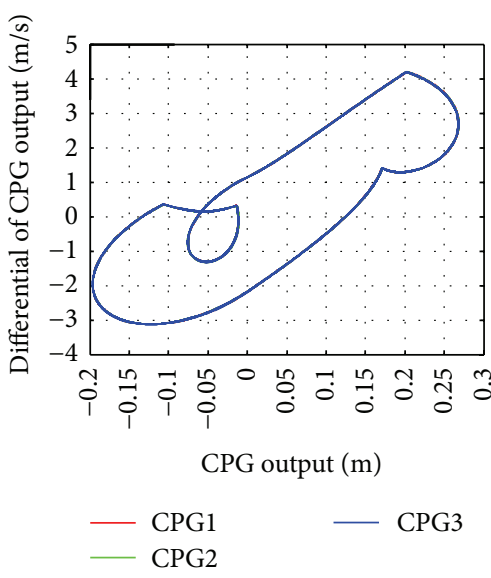

(b)

Figure 2: Outputs and phase diagrams of three CPGs: (a) the CPGs outputs and (b) the phase diagrams of these CPGs.

TABLE 1: Octopus arm simulation parameters.

\begin{tabular}{ll}
\hline Parameter & Implication \\
\hline$N$ & $\begin{array}{l}(N-1) \text { is the number of segments } \\
\text { constructing the arm }\end{array}$ \\
\hline$g$ & Gravitational acceleration $\left[\mathrm{m} / \mathrm{s}^{2}\right]$ \\
\hline water_sw & $\begin{array}{l}\text { Specific weight of the medium (seawater, in } \\
\text { this case) }\left[\mathrm{kg} / \mathrm{m}^{3}\right]\end{array}$ \\
\hline arm_sw & Specific weight of the octopus arm $\left[\mathrm{kg} / \mathrm{m}^{3}\right]$ \\
\hline passive_elasticity & $\begin{array}{l}\text { Ratio between maximal and minimal spring } \\
\text { constants }\end{array}$ \\
\hline sim_time & Simulation time [s] \\
\hline delta_t & Time resolution for simulation $[\mathrm{s}]$ \\
\hline act_interval & Interval between possible activation changes [s] \\
\hline$n \_a c t \_t y p e s$ & Number of available activations \\
\hline slow_base & $\begin{array}{l}\text { Force proportional to base speed, resisting its } \\
\text { rotation }\end{array}$ \\
\hline Activation & $\begin{array}{l}\text { Declaration of an activation followed by a serial } \\
\text { number, base rotation moment, and activation } \\
\text { values for each spring }\end{array}$ \\
\hline
\end{tabular}

2.4. Model of Interaction between the CPG and the Octopus Arm. The coupled CPG model [21,22] can be described by

$$
\begin{aligned}
& T_{r} \dot{x_{1}}+x_{1}=-d x_{4}-w g\left(x_{2}\right)-w g\left(x_{3}\right)+e, \\
& T_{a} \dot{x_{4}}+x_{4}=g\left(x_{1}\right), \\
& T_{r} \dot{x_{2}}+x_{2}=-d x_{5}-w g\left(x_{1}\right)-w g\left(x_{3}\right)+e, \\
& T_{a} \dot{x_{5}}+x_{5}=g\left(x_{2}\right), \\
& T_{r} \dot{x_{3}}+x_{3}=-d x_{6}-w g\left(x_{1}\right)-w g\left(x_{2}\right)+e, \\
& T_{a} \dot{x_{6}}+x_{6}=g\left(x_{3}\right),
\end{aligned}
$$

$$
\begin{aligned}
& y_{1}=g\left(x_{1}\right), \\
& y_{2}=g\left(x_{2}\right), \\
& y_{3}=g\left(x_{3}\right) .
\end{aligned}
$$

The function $g(\cdot)$ is a piecewise linear function defined by $g(x)=\max (o, x)$, which represents a threshold property of the neurons. These variables $x_{1}, x_{2}$, and $x_{3}$ represent the membrane potential. Self-inhibitory inputs $x_{4}, x_{5}$, and $x_{6}$ represent adaptation or fatigue property that ubiquitously exists in real neurons. The parameter $e$ denotes the tonic input and determines the amplitude of CPG output. Parameters $w$ and $d$ represent the strength of mutual and self-inhibition, respectively; parameters $T_{r}$ and $T_{a}$ are the time constants that determine the reaction times of variables $x_{1}, x_{2}$, and $x_{3}$ and $x_{4}, x_{5}$, and $x_{6}$. In other words, parameters $T_{r}$ and $T_{a}$ determine the frequency of CPG output. Three variables $y_{1}$, $y_{2}$, and $y_{3}$ represent the output of three CPGs, respectively.

The fundamental values of these CPG parameters are set as $T_{r}=0.1 \mathrm{~s}, T_{a}=0.2 \mathrm{~s}, d=4.5, w=4.5$, and $e=1$. And the initial values are set as $\left[\begin{array}{llllll}0.1 & 0 & 0 & 0 & 0 & 0.1\end{array}\right]$. Then the CPGs' output and phase diagrams are obtained, as shown in Figure 2.

In Figure 2(a), there is a uniform phase difference among three CPGs and the three phase diagrams have the same shape and they overlap each other. And the output of CPG approximates the overall shape of the electromyograms (EMG) activation measured in an octopus arm during reaching movements $[23,24]$. EMG recorded from arm muscles showed that the octopus arm movement is associated with a propagating wave of muscle activation [24]. Therefore, each CPG output can be treated as the activation wave.

Yekutieli et al. [11] showed that the mechanism for bend propagation is a stiffening wave caused by muscle activation pattern. Therefore, it is reasonable that one-cycle outputs of three coupled CPGs treated as activation wave are added to the ventral, dorsal, and transversal sides of the octopus arm, 




Three coupled CPGs

Octopus arm model

FIGURE 3: Model of interaction between the CPGs and the octopus arm.

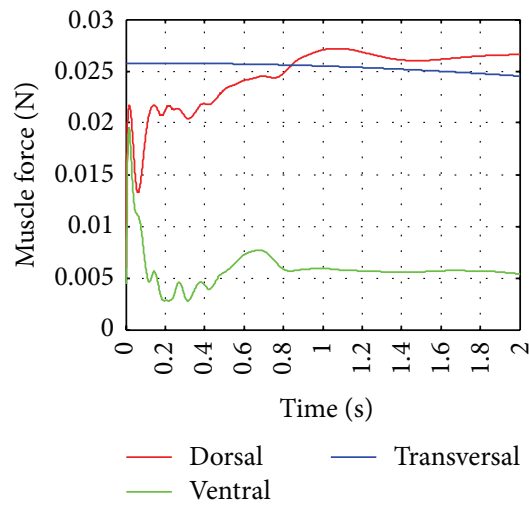

(a)

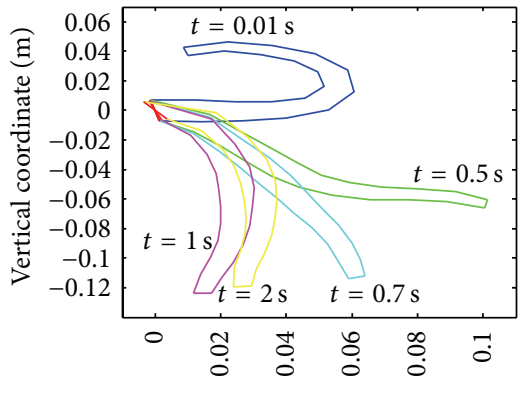

Horizontal coordinate $(\mathrm{m})$

(b)

FIGURE 4: Muscles forces of the first octopus arm segment and a sequence of octopus arm movements: (a) muscles forces of the first segment and (b) a sequence of octopus arm movements.

respectively. And the model of interaction between the CPGs and the octopus arm is shown in Figure 3.

In Figure 3, the left part is the control block diagram of the three coupled CPGs [22], and the right one is the control block diagram of the octopus arm model by using Laplace transform. Parameters $F_{d}, F_{v}$, and $F_{t}$ denote the force generated by the arm muscles of the ventral, dorsal, and transversal sides, respectively.

The octopus arm parameters are set as $g=9.8 \mathrm{~m} / \mathrm{s}^{2}$, arm_sw $=1200 \mathrm{~kg} / \mathrm{m}^{3}$, water_sw $=1025 \mathrm{~kg} / \mathrm{m}^{3}$, muscle_strength $=5000 \mathrm{~N} / \mathrm{m}^{2}$, passive_elasticity $=0.03$, sim_time $=2 \mathrm{~s}$, delta_t
$=0.001 \mathrm{~s}$, slow_base $=0.37$, act_interval $=0.5 \mathrm{~s}$, and $n_{-}$act_types $=5$. Then the arm muscles' forces are obtained, as shown in Figure 4(a). And a sequence of octopus arm movements is shown in Figure 4(b). In the sequence of octopus arm motion, the times are selected as $0.01 \mathrm{~s}, 0.5 \mathrm{~s}, 0.7 \mathrm{~s}, 1 \mathrm{~s}$, and $2 \mathrm{~s}$.

Because the muscles forces of other segments are similar to the ones of the first segment, the diagram of the first segment is shown only. In Figure 4(a), the forces generated by the dorsal and ventral sides are symmetrical and the force of the transversal side change little all the simulation time. Moreover, the movements in Figure 4(b) can mimic 

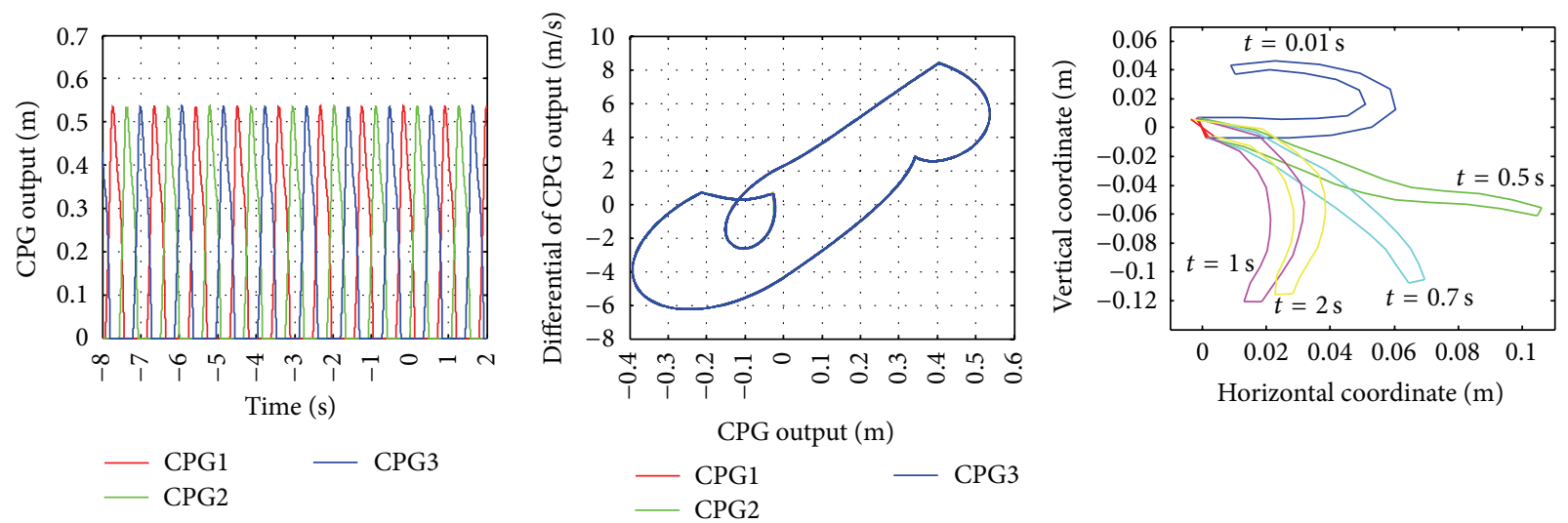

(a)
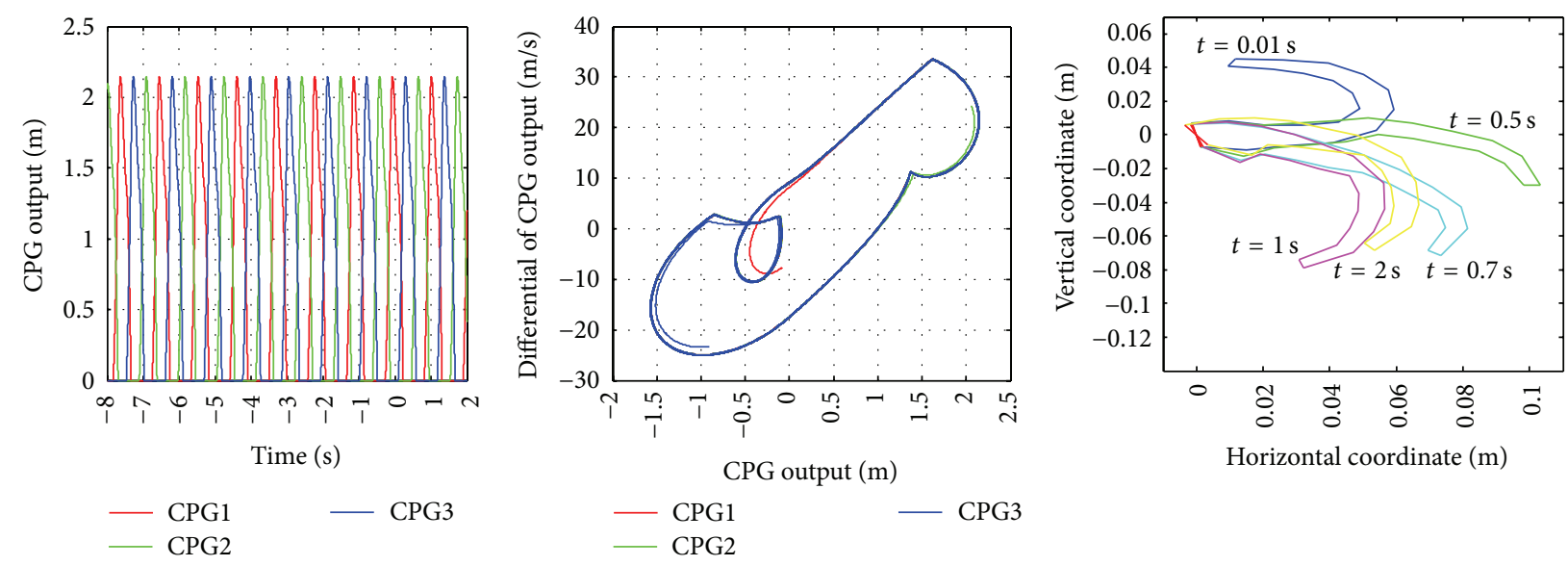

(b)
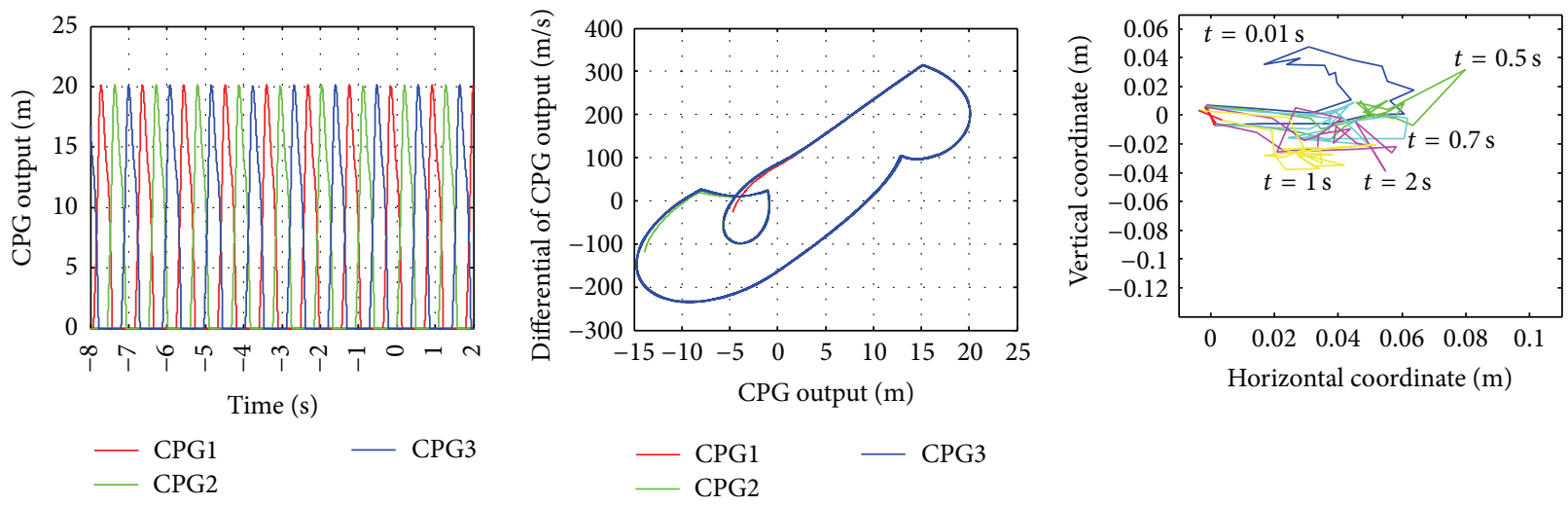

(c)

FIGURE 5: Outputs and phase diagrams of three coupled CPGs and a sequence of octopus arm movements with the parameter $e$ (from left to right): (a) $e=2$, (b) $e=8$, and (c) $e=75$.

the octopus movements. This simulation shows the mechanism for octopus movements: a stiffening wave caused by a symmetrical muscle activation pattern propagates along the arm and propels the octopus movements [11].

\section{Simulation}

Now the effects of octopus arm movements are discussed by simulation when the parameters of CPG are changed.
3.1. Effects of Octopus Arm Motion with Parameters $e$ and $T_{r}$. In this simulation, the value of parameter $e$ is varied in the interval $(0,100]$ in step of 1 . The outputs and phase diagrams of three coupled CPGs are obtained and a sequence of octopus arm movements is shown in Figure 5. When $e \in$ $(0,4)$, the shapes of three CPGs' phase diagrams are all the limit cycles and the simulation can mimic the movements of the octopus. The typical diagram is shown in Figure 5(a). While $e \geq 4$, there exists difference in the phase diagrams 

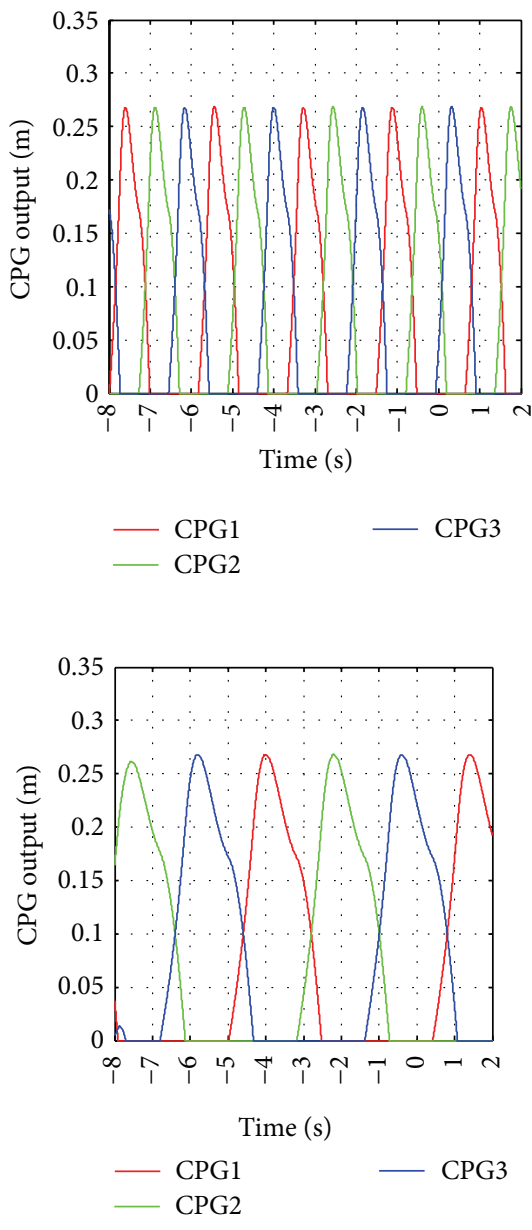
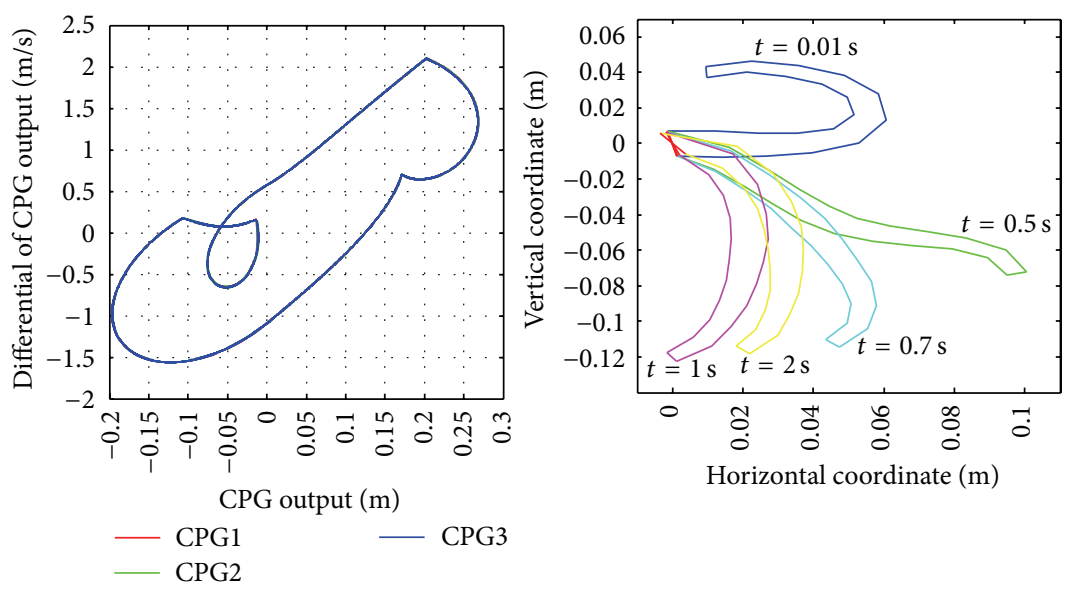

(a)
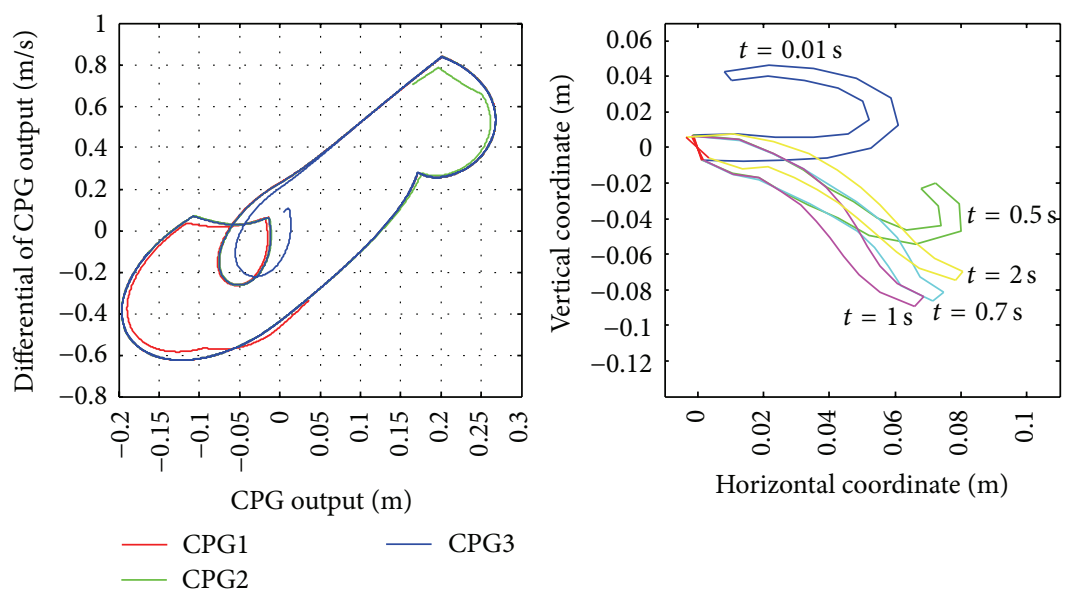

(b)

Figure 6: Outputs and phase diagrams of three coupled CPGs and a sequence of octopus arm movements with the parameter $T_{r}$ (from left to right): (a) $T_{r}=0.2$ and (b) $T_{r}=0.5$.

of the three coupled CPGs and they do not overlap. The tip of octopus arm is not as straight as one in Figure 5(a). The typical diagram is shown in Figure 5(b). With the increase of the parameter $e$, the phase diagrams of the three CPGs become the limit cycles again. However, the degree of overlap decreases and the difference of the phase diagrams among three coupled CPGs becomes larger when $e \geq 53$. And the sequence of octopus arm movements is in disorder. The typical diagram is shown in Figure 5(c).

Here the effects of octopus arm are studied when the parameter $T_{r}$ is changed and $e=1$. The value of parameter $T_{r}$ is varied in the interval $(0,1]$ in step of 0.1 . When $T_{r} \in(0,0.2]$, the phase diagrams of the three CPGs are all the limit cycles and they overlap completely. The typical diagram is shown in Figure 6(a). While $T_{r} \geq 0.3$, there exists difference of the phase diagrams among the three coupled CPGs. And the movements of the octopus are changed. The typical diagram is shown in Figure 6(b). However, the difference of the phase diagrams among the three coupled CPGs becomes larger and the limit cycles are broken when $T_{r}=1$. Then it cannot generate sustaining rhythmic motion.
3.2. Effects of Octopus Arm Motion with Parameters $d$ and $w$. The effects of octopus arm are discussed when parameters $d$ and $w$ are changed, and other parameters are set as $e=1$ and $T_{r}=0.1$. The values of parameters $d$ and $w$ are varied in the interval $(0,200]$ in step of 1 . At the beginning, the phase diagrams of the CPGs are not the limit cycles and the movements cannot be continuous. When $d=w=3$, the phase diagrams of the three CPGs are the limit cycles and the shapes are circles. But the three circles do not overlap each other. The movements of the octopus are different from the ones in Figure 6(a). The typical diagram is shown in Figure 7(a). When $d \geq 4$ and $w \geq 4$, the diagrams are similar to ones in Figure 6(a). The typical diagram is shown in Figure $7(\mathrm{~b})$. With the increase of parameters $d$ and $w$, the phase diagram of each CPG gradually changes to be a type of the limit cycle whose shape is different from the one in Figure 6(b) when $d \geq 12$ and $w \geq 12$. The typical diagram is shown in Figure 7(c).

The amplitude of CPG is proportional to the tonic input $e$, and the frequency of the limit cycle oscillation is proportional to $1 / T_{r}$ [22]. From the above simulation, the amplitude 

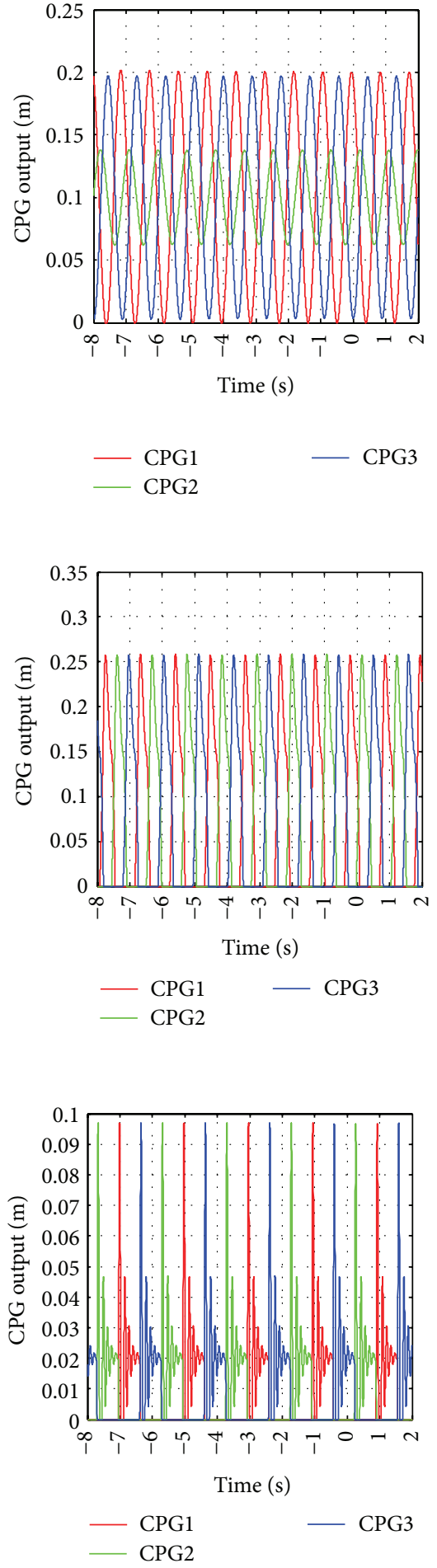

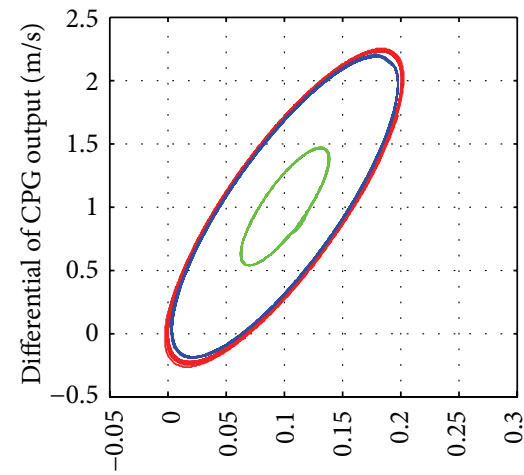

$$
\begin{aligned}
\text { CPG output (m) } \\
- \text { CPG1 } \\
- \text { CPG2 }
\end{aligned}
$$

(a)

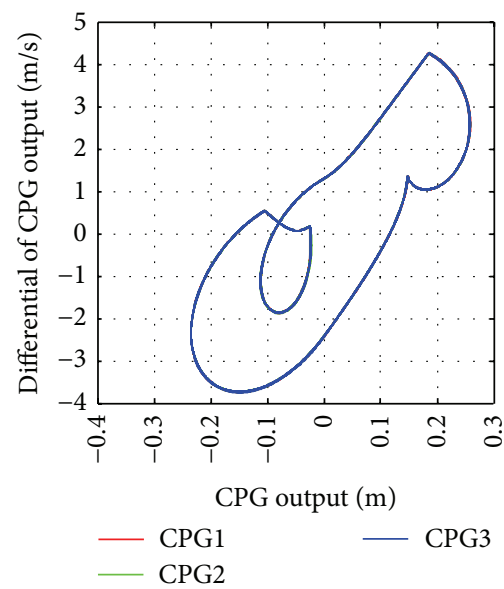

(b)

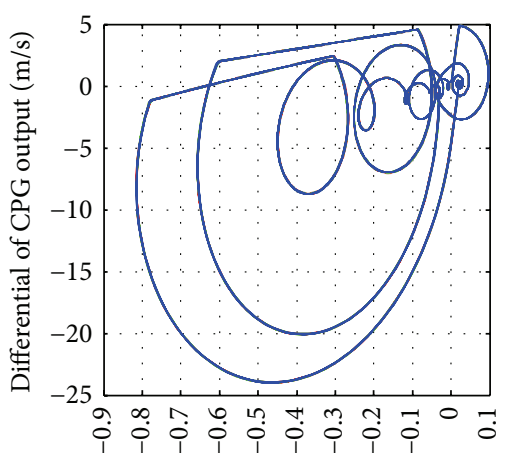

CPG output (m)



(c)

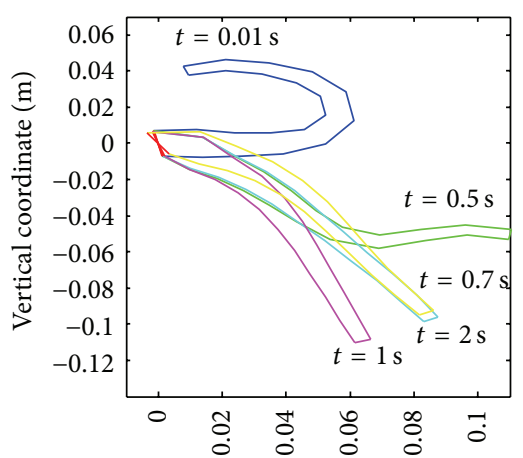

Horizontal coordinate (m)

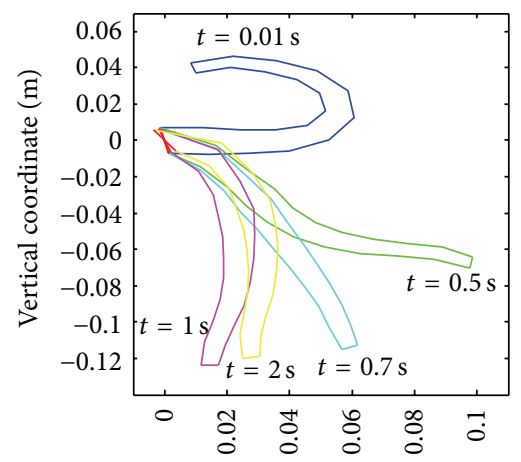

Horizontal coordinate (m)

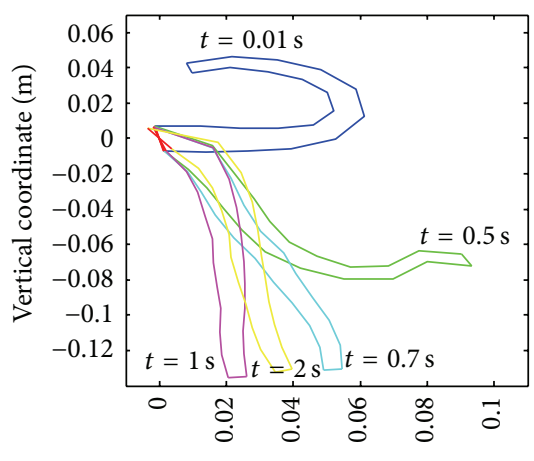

Horizontal coordinate $(\mathrm{m})$

FIGURE 7: Outputs and phase diagrams of three coupled CPGs and a sequence of octopus arm movements with parameters $d$ and $w$ (from left to right): (a) $d=w=3$, (b) $d=w=5$, and (c) $d=w=48$. 


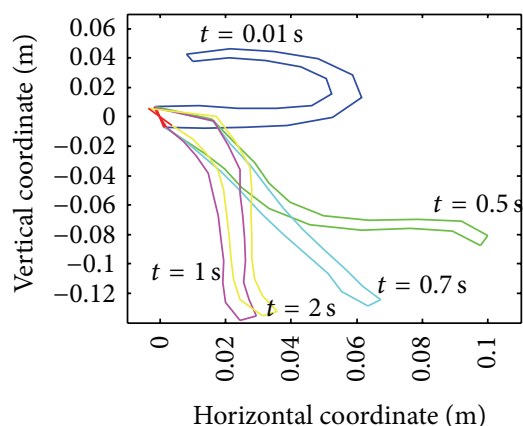

(a)

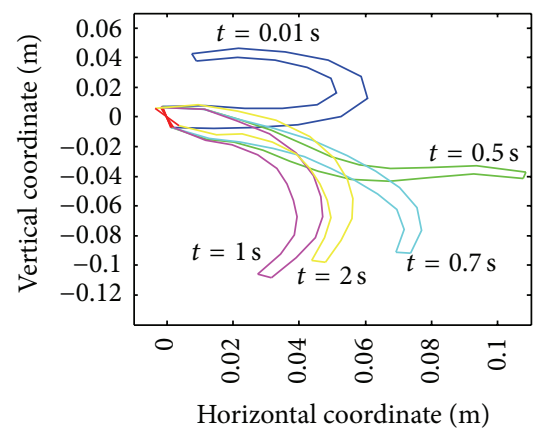

(b)

FIGURE 8: A sequence of octopus arm movements: (a) the third CPG for ventral side and the first CPG for transversal side and (b) the third CPG for dorsal side and the second CPG for transversal side.

and frequency should be in an allowable range. The larger amplitude leads the octopus arm to be disorder. The smaller frequency causes the limit cycle to be broken and the octopus movement cannot be sustaining. However, parameters $d$ and $w$ also affect the shapes of the CPGs' phase diagrams which correspond to different octopus movements.

\section{Discussion and Conclusion}

In Figures 5-7, the octopus arm movements are determined by the shape and overlap degree of the three CPGs' phase diagrams. Different shapes of the phase diagrams correspond to different motion types, as shown in Figures $7(\mathrm{a})-7(\mathrm{c})$. Moreover, analogical shape of the phase diagram leads to similar movement of the octopus arm, as shown in Figures 5(a), 6(a), and 7(b)-7(c). Although the shapes of the three coupled CPGs are similar, the difference among them leads to different motion, as shown in Figures 5(a)-5(b) and 6(a)-6(b). The activation time also affects the octopus arm movement. The octopus arm locates in different position with different time.

The sequence of the three coupled CPGs also affects the octopus arm movements. Taking Figure 5(a) as an example, when changing the sequence of the three coupled CPGs, the octopus arm movements are shown in Figure 8. In Figure 8(a), the third CPG is for ventral side and the first CPG is for transversal one. In Figure 8(b), the third CPG is for dorsal side and the second CPG is for transversal one. Then the octopus movements are different from the ones in Figure 5(a).

Sumbre et al. [13] showed that the octopus reduces the complexity of controlling the flexible appendage by using highly stereotypical movements and there appears to be an underlying motor program embedded in the neuromuscular system of the arm. From simulations above, some locomotion modes are suggested to be embedded in the neuromuscular system of the arm. And the octopus arm movements can be achieved by modulating the parameters of the CPGs. And the simulation results enhance and improve the conclusion in [13]. The results are beneficial for researchers to understand the octopus movement further.
The octopus arm can apply force with the sole use of muscles without any rigid skeletal support. The biomechanical attributes of such an arm enable it to perform tasks no skeletal arm can perform. Hence, a robotic implementation of an octopus arm with a real-time learning control mechanism will yield a highly versatile application. And it is the direction of the future works.

\section{Conflict of Interests}

The authors declare that there is no conflict of interests regarding the publication of this paper.

\section{Acknowledgments}

The work is supported by Project of Shandong Province Higher Educational Science and Technology Program, China (Grant no. J13LN04), and Taian Science and Technology Development Program, China (Grant no. 201430774).

\section{References}

[1] S. Kim, C. Laschi, and B. Trimmer, "Soft robotics: a bioinspired evolution in robotics," Trends in Biotechnology, vol. 31, no. 5, pp. 287-294, 2013.

[2] C. Laschi, M. Cianchetti, B. Mazzolai, L. Margheri, M. Follador, and P. Dario, "Soft robot arm inspired by the octopus," Advanced Robotics, vol. 26, no. 7, pp. 709-727, 2012.

[3] L. Margheri, C. Laschi, and B. Mazzolai, "Soft robotic arm inspired by the octopus: I. From biological functions to artificial requirements," Bioinspiration \& Biomimetics, vol. 7, no. 2, Article ID 025004, 2012.

[4] W. M. Kier and M. P. Stella, "The arrangement and function of octopus arm musculature and connective tissue," Journal of Morphology, vol. 268, no. 10, pp. 831-843, 2007.

[5] R. Kang, D. T. Branson, E. Guglielmino, and D. G. Caldwell, "Dynamic modeling and control of an octopus inspired multiple continuum arm robot," Computers and Mathematics with Applications, vol. 64, no. 5, pp. 1004-1016, 2012.

[6] T. Zheng, D. T. Branson, E. Guglielmino, and D. G. Caldwell, "A 3D dynamic model for continuum robots inspired by an 
octopus arm," in Proceedings of the 2011 IEEE International Conference on Robotics and Automation (ICRA '11), pp. 36523657, IEEE, Shanghai, China, May 2011.

[7] T. Zheng, D. T. Branson, R. Kang et al., "Dynamic continuum arm model for use with underwater robotic manipulators inspired by octopus vulgaris," in Proceedings of the IEEE International Conference on Robotics and Automation, pp. 5289-5294, Saint Paul, Minn, USA, 2012.

[8] F. Renda, M. Cianchetti, M. Giorelli, A. Arienti, and C. Laschi, "A 3D steady-state model of a tendon-driven continuum soft manipulator inspired by the octopus arm," Bioinspiration and Biomimetics, vol. 7, no. 2, Article ID 025006, 2012.

[9] K. Nakajima, H. Hauser, R. Kang, E. Guglielmino, D. G. Caldwell, and R. Pfeifer, "A soft body as a reservoir: case studies in a dynamic model of octopus-inspired soft robotic arm (11916 words)," Frontiers in Computational Neuroscience, vol. 7, article 91, 2013.

[10] Y. Yekutieli, R. Sagiv-Zohar, R. Aharonov, Y. Engel, B. Hochner, and T. Flash, "Dynamic model of the octopus arm. I. Biomechanics of the octopus reaching movement," Journal of Neurophysiology, vol. 94, no. 2, pp. 1443-1458, 2005.

[11] Y. Yekutieli, R. Sagiv-Zohar, B. Hochner, and T. Flash, "Dynamic model of the octopus arm. II. Control of reaching movements," Journal of Neurophysiology, vol. 94, no. 2, pp. 1459-1468, 2005.

[12] Y. Gutfreund, "Patterns of arm muscle activation involved in octopus reaching movements," Journal of Neuroscience, vol. 18, no. 15 , pp. 5976-5987, 1998.

[13] G. Sumbre, Y. Gutfreund, G. Fiorito, T. Flash, and B. Hochner, "Control of octopus arm extension by a peripheral motor program," Science, vol. 293, no. 5536, pp. 1845-1848, 2001.

[14] A. J. Ijspeert, "Central pattern generators for locomotion control in animals and robots: a review," Neural Networks, vol. 21, no. 4, pp. 642-653, 2008.

[15] R. M. Harris-Warrick, "Neuromodulation and flexibility in central pattern generator networks," Current Opinion in Neurobiology, vol. 21, no. 5, pp. 685-692, 2011.

[16] C. J. Liu, D. W. Wang, and Q. J. Chen, "Locomotion control of quadruped robots based on workspace trajectory modulations," International Journal of Robotics and Automation, vol. 27, no. 4, pp. 345-354, 2012.

[17] K. Matsuoka, "Sustained oscillations generated by mutually inhibiting neurons with adaptation," Biological Cybernetics, vol. 52, no. 6, pp. 367-376, 1985.

[18] K. Matsuoka, "Mechanisms of frequency and pattern control in the neural rhythm generators," Biological Cybernetics, vol. 56, no. 5-6, pp. 345-353, 1987.

[19] P. Wawrzyński and A. K. Tanwani, "Autonomous reinforcement learning with experience replay," Neural Networks, vol. 41, pp. 156-167, 2013.

[20] T. D. Frank, T. D. Gifford, and S. Chiangga, "Minimalistic model for navigation of mobile robots around obstacles based on complex-number calculus and inspired by human navigation behavior," Mathematics and Computers in Simulation, vol. 97, pp. 108-122, 2014.

[21] Q. Lu and J. Tian, "Synchronization and stochastic resonance of the small-world neural network based on the CPG," Cognitive Neurodynamics, vol. 8, pp. 217-226, 2014.

[22] K. Matsuoka, "Analysis of a neural oscillator," Biological Cybernetics, vol. 104, no. 4-5, pp. 297-304, 2011.
[23] Y. Gutfreund, T. Flash, Y. Yarom, G. Fiorito, I. Segev, and B. Hochner, "Organization of octopus arm movements: a model system for studying the control of flexible arms," Journal of Neuroscience, vol. 16, no. 22, pp. 7297-7307, 1996.

[24] Y. Gutfreund, T. Flash, G. Fiorito, and et al, "Patterns of arm muscle activation involved in octopus reaching movements," Journal of Neuroscience, vol. 18, no. 15, pp. 5976-5987, 1998. 

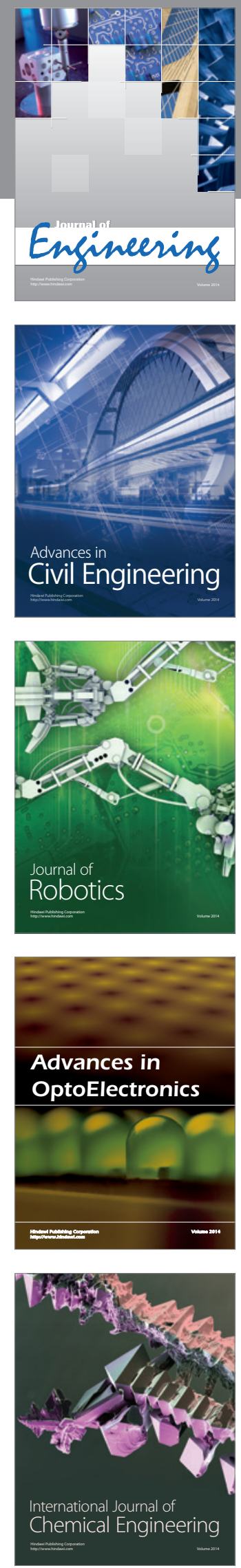

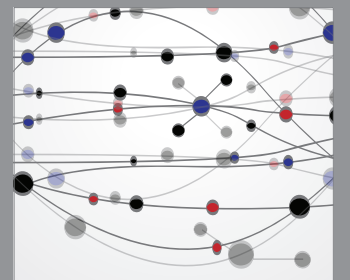

The Scientific World Journal
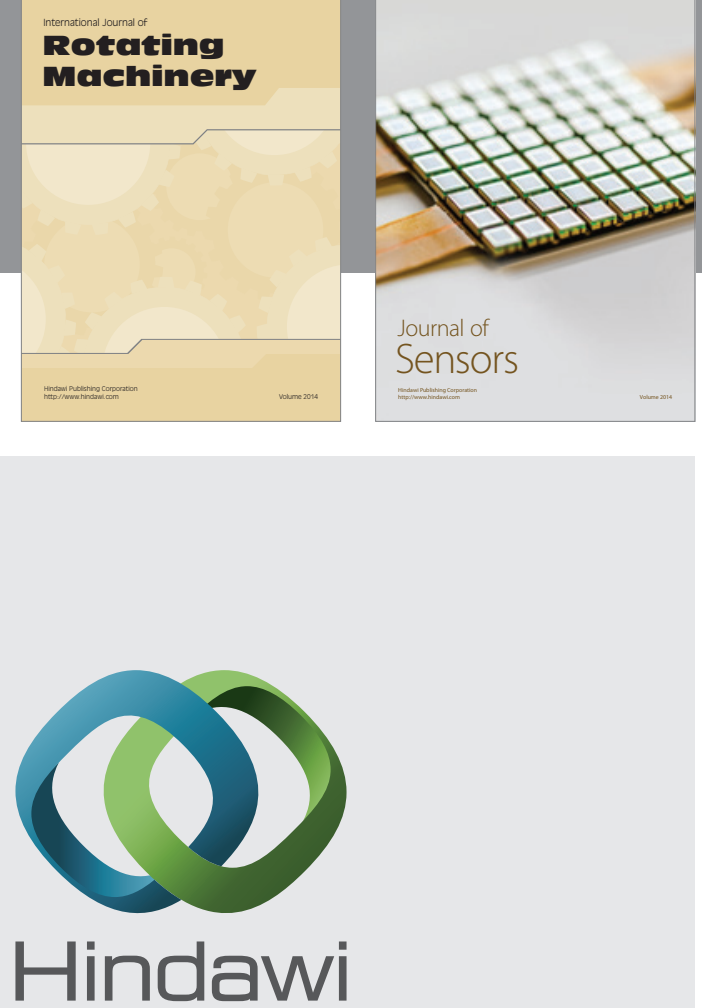

Submit your manuscripts at http://www.hindawi.com
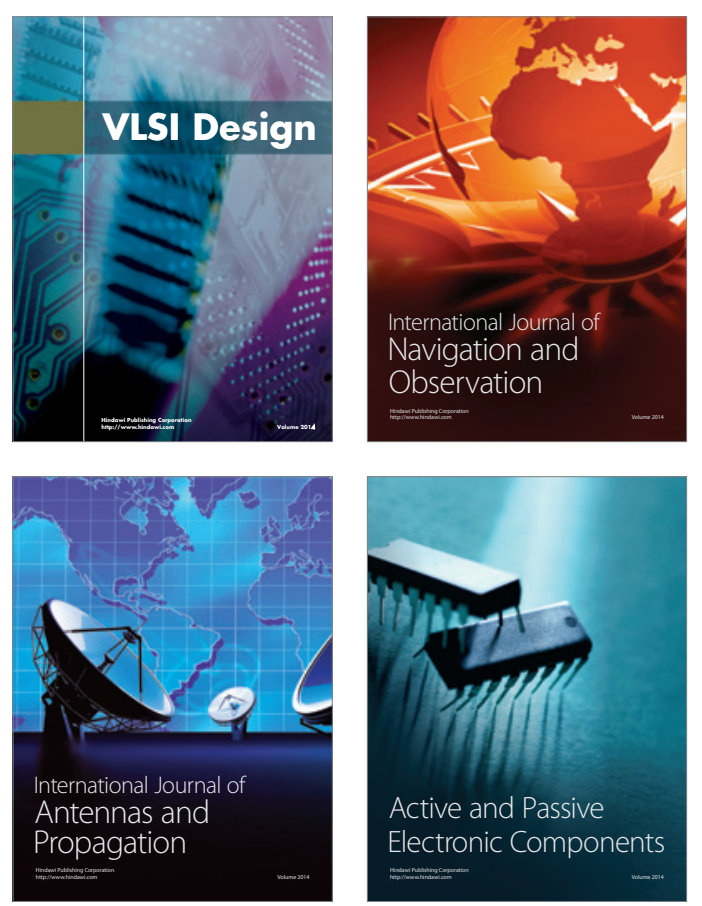
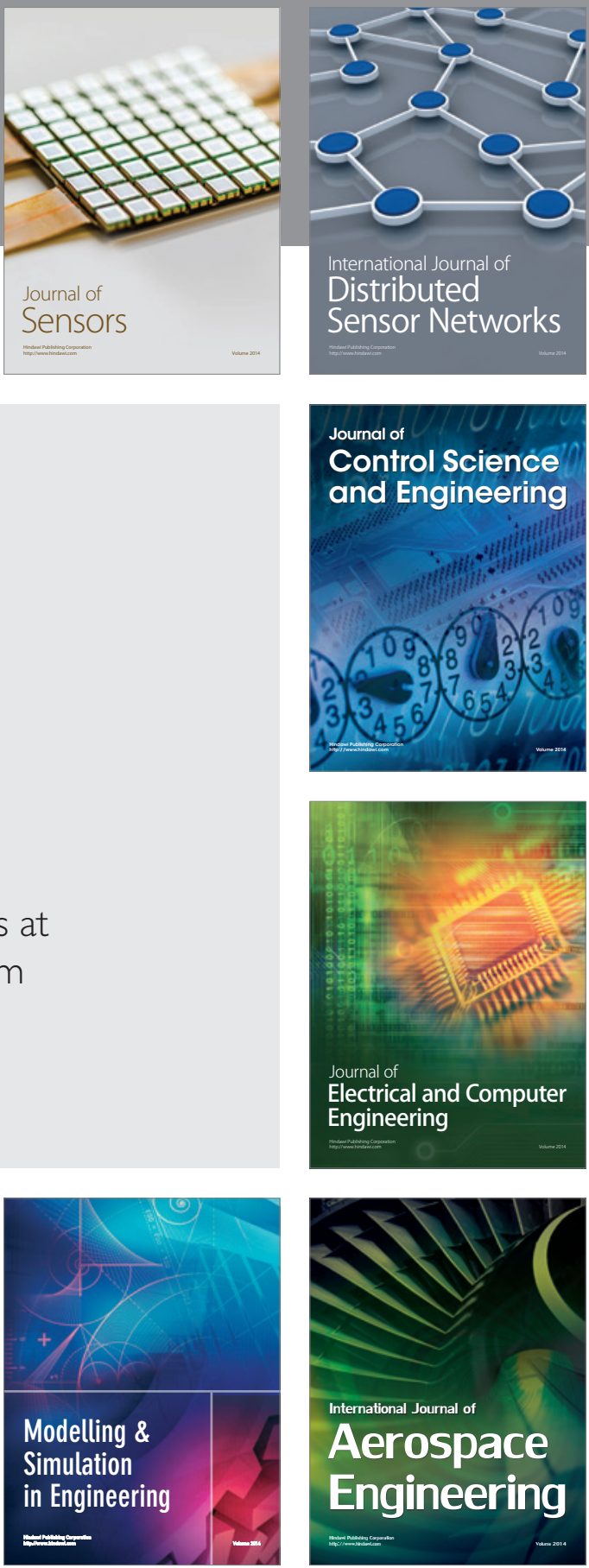

Journal of

Control Science

and Engineering
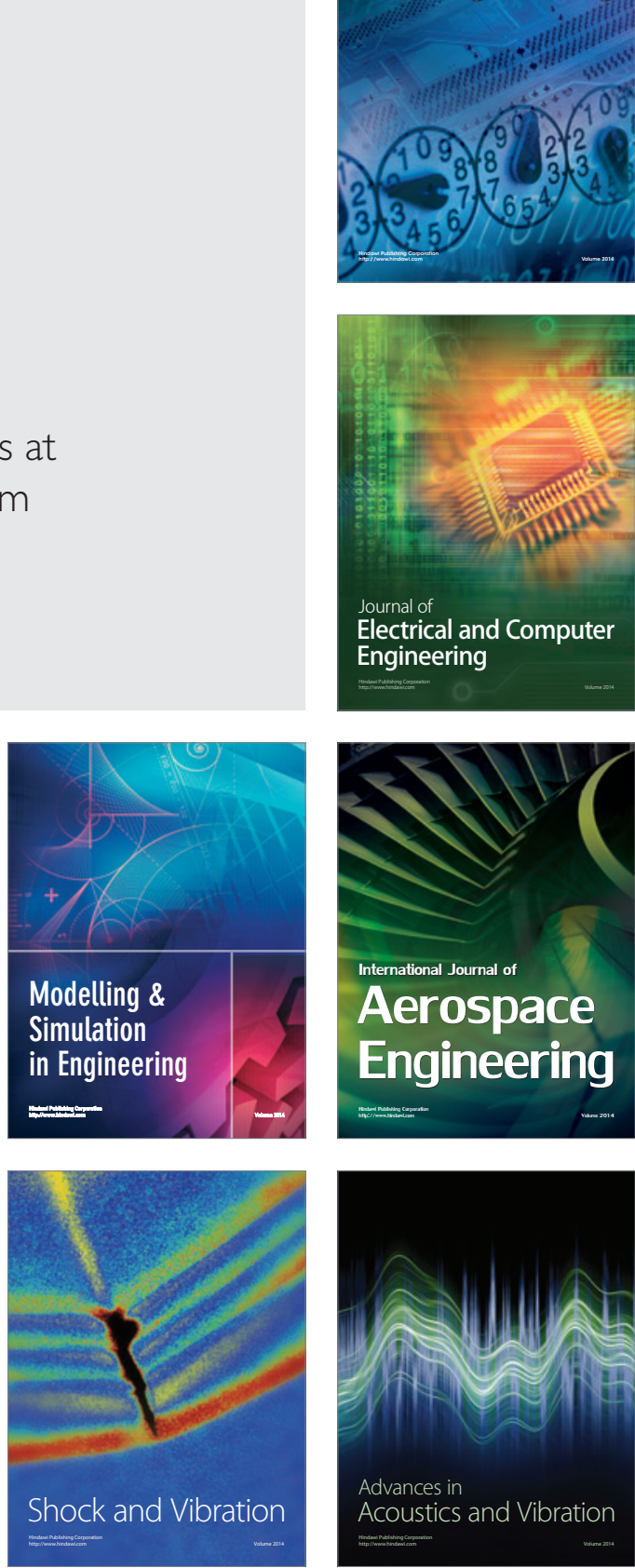